\title{
Deterministic conversion of uncertain manpower planning optimization problem
}

\author{
Bo Li, Yuanguo Zhu, Yufei Sun, Grace Aw and Kok Lay Teo
}

\begin{abstract}
Manpower planning is a very important component of human resource management. However, there are many indeterminate factors that should be taken into consideration in manpower planning. For example, the decision of employees to quit the job is determined by their preference, which is beyond the control of human resource department. It can be realistically modeled as a random variable when the historical data of quitting rate is large enough. Otherwise, it can only be regarded as an uncertain variable when the historical data is inadequate. In this paper, we discuss a manpower planning optimization problem for a manufacturing company with hierarchical system, where the quitting rate of employees is modeled as an uncertain variable. First, we formulate a mathematical model for this uncertain manpower planning optimization problem, where the influence on the production outputs by employees is taken into consideration. Second, we present a deterministic conversion method to transfor$m$ this uncertain manpower planning optimization problem into an equivalent deterministic discrete-time optimization problem. It is further converted into an equivalent linear programming model with an equality constraint and an inequality constraint. Finally, we use the real data from Singapore, Denmark and China to carry out a numerical simulation and make a comparison with the results obtained based on stochastic model to show the advantages of our method.
\end{abstract}

Index Terms-Manpower planning, quitting rate, uncertainty theory, uncertain variable, linear programming.

\section{INTRODUCTION}

W ITH the rapid development of economy and increasing globalization, manpower planning has become a critical issue for human resource department in today's competitive world, especially for the international corporations and large organizations. It requires to develop an optimal management strategy to match the requirement of the staffs and the available positions for achieving specific goals.

However, there are many indeterminate factors that should be taken into consideration in manpower planning, such as labor demand, working life and economic environment. At present, many different stochastic manpower planning models

Bo Li is with the School of Applied Mathematics, Nanjing University of Finance and Economics, Nanjing 210023, China (email: libnust@ 163.com).

Yuanguo Zhu is with the School of Science, Nanjing University of Science and Technology, Nanjing 210094, China (email: ygzhu@njust.edu.cn).

Yufei Sun is with the Department of Mathematics and Statistics, Curtin University, Perth 6102, Australia and the School of Mathematical Sciences, Chongqing Normal University, Chongqing 401331, China (email: rainfei0530@hotmail.com).

Grace Aw is with the Department of Mathematics and Statistics, Curtin University, Perth 6102, Australia (email: grace.aw.ee.ling@gmail.com).

Kok Lay Teo is with the Department of Mathematics and Statistics, Curtin University, Perth 6102, Australia and the Coordinated Innovation Center for Computable Modeling in Management Science, Tianjin University of Finance and Economics, Tianjin 300222, China (email: K.L.Teo@ curtin.edu.au).

*Corresponding author: Bo Li (email: libnust@ 163.com). have been established and discussed in the literature. For instance, Chattopadhyay and Gupta [2] developed a stochastic manpower planning model under the set up, where the survival rates and the number of workers at different ages are treated as random variables. Yan et al. [18] discussed two long-term stochastic demand planning models for air cargo terminal manpower supply planning in long-term operations, where the labor demand is described as a random variable. Young and Vassiliou [23] considered a non-linear stochastic model of hierarchically structured management staffs in commercial and industrial organizations, where the promotion of employees is modeled as a random variable.

However, for most of the related literature, the effect of the optimal planning strategy on the production outputs has not been taken into full consideration. In 2016, Sun et al. [16] constructed a new model of a manpower planning optimization problem for a manufacturing company with different types of employees, where the types of workers have direct influence on the production outputs, and the quitting rate of employees was assumed as a random variable with known expected value and variance. Then, the manpower planning optimization problem was formulated as a stochastic discrete time optimization model.

When we need to describe a subjective imprecise quantity, the concept of belief degree will be used. In order to deal with personal belief degrees, an uncertainty theory was introduced by Liu [10] in 2007 and refined by Liu [14] in 2010 based on normality, duality, subadditivity and product axioms. After that, uncertainty theory has been investigated by many researchers. Sheng and Kar [15] provided a new formula using inverse uncertainty distribution to describe the moment of uncertain variable. Yao [20] proposed a formula to calculate the variance of an uncertain variable via the inverse uncertainty distribution. Yao and Li [22] presented a kind of uncertain process, called uncertain alternating renewal process and developed an uncertain renewal theory. Zhu [24] introduced and dealt with an uncertain optimal control problem with application to a portfolio selection model. Up to now, uncertainty theory has become a branch of axiomatic mathematics and contains many topics, such as uncertain programming (Gao [8], Liu [13]), uncertain process (Chen [3], Liu [11]), uncertain finance (Chen and Gao [4], Chen et al. [6]) and uncertain differential equation (Chen and Liu [5], Yao et al. [21]).

As mentioned above, the quitting rate of employees for a manufacturing company is usually uncertain in nature, which should be taken into consideration during the decision making process in human resource management. Hence, the manpower 
planning optimization problem under uncertain environment is as important as in stochastic environment. In this paper, we consider a manpower planning optimization problem for a manufacturing company with hierarchical system under uncertain environment, where the quitting rate is modeled as an uncertain variable. We shall compare our results with those obtained using the stochastic model proposed in Sun et al. [16].

The rest of this paper is organized as follows. Some basic concepts in uncertain theory are reviewed in Section II. In Section III, the manpower planning optimization problem is formulated as an uncertain discrete time optimization model. In Section IV, we transform it into a deterministic discrete time optimization model and propose a method to solve it. Based on the real data from Singapore, Denmark and China, a numerical simulation is carried out in Section V. The last section gives a conclusion.

\section{PREliminary}

For the formulation and discussion of the manpower planning optimization problem under uncertain environment, many basic concepts, such as uncertain variable, uncertainty distribution and uncertain expected value, in uncertainty theory will be used in Section III. Thus, they will be introduced in this section.

Let $\Gamma$ be a nonempty set, and $\mathcal{L}$ a $\sigma$-algebra over $\Gamma$. Each element $\Lambda \in \mathcal{L}$ is called an event. A set function $\mathcal{M}$ defined on the $\sigma$-algebra over $\mathcal{L}$ is called an uncertain measure if it satisfies the following axioms: (normality axiom) $\mathcal{M}\{\Gamma\}=1$ for the universal set $\Gamma$; (duality axiom) $\mathcal{M}\{\Lambda\}+\mathcal{M}\left\{\Lambda^{c}\right\}=1$ for any event $\Lambda$; (subadditivity axiom) $\mathcal{M}\left\{\bigcup_{i=1}^{\infty} \Lambda_{i}\right\} \leq \sum_{i=1}^{\infty} \mathcal{M}\left\{\Lambda_{i}\right\}$ for every countable sequence of events $\Lambda_{1}, \Lambda_{2}, \cdots$.

The triplet $(\Gamma, \mathcal{L}, \mathcal{M})$ is called an uncertainty space. A product uncertain measure $\mathcal{M}$ is defined by Liu [12] to produce an uncertain measure of compound event: (product axiom) Let $\left(\Gamma_{k}, \mathcal{L}_{k}, \mathcal{M}_{k}\right)$ be uncertainty spaces for $k=1,2, \cdots$. Then the product uncertain measure $\mathcal{M}$ is an uncertain measure satisfying

$$
\mathcal{M}\left\{\prod_{k=1}^{\infty} \Lambda_{k}\right\}=\bigwedge_{k=1}^{\infty} \mathcal{M}_{k}\left\{\Lambda_{k}\right\}
$$

where $\Lambda_{k}$ are arbitrarily chosen events from $\mathcal{L}_{k}$ for $k=$ $1,2, \cdots$, respectively.

For modeling the quantities under uncertain environment, a concept of uncertain variable is defined by Liu [10] as a function $\xi$ from an uncertainty space $(\Gamma, \mathcal{L}, \mathcal{M})$ to the set of real numbers such that for any Borel set of real numbers, the set $\{\xi \in B\}=\{\gamma \in \Gamma \mid \xi(\gamma) \in B\}$ is an event. In order to describe uncertain variable in practice, the uncertainty distribution $\Phi$ of an uncertain variable $\xi$ is defined by $\Phi(x)=$ $\mathcal{M}\{\xi \leq x\}$, for any real number $x$.

An uncertain variable $\xi$ is called normal if it has a normal uncertainty distribution

$$
\Phi(x)=\left(1+\exp \left(\frac{\pi(e-x)}{\sqrt{3} \sigma}\right)\right)^{-1}, \quad x \in \Re
$$

denoted by $\xi \sim \mathcal{N}(e, \sigma)$, where $e$ and $\sigma$ are real numbers with $\sigma>0$.
To describe the average value of an uncertain variable $\xi$, the concept of expected value is defined in Liu [10] as

$$
E[\xi]=\int_{0}^{+\infty} \mathcal{M}\{\xi \geq x\} \mathrm{d} x-\int_{-\infty}^{0} \mathcal{M}\{\xi \leq x\} \mathrm{d} x
$$

provided that at least one of the two integrals is finite. The variance of $\xi$ is defined as

$$
\operatorname{Var}[\xi]=E\left[(\xi-E[\xi])^{2}\right] .
$$

Let $\xi$ and $\eta$ be independent uncertain variables with finite expected values. Then, for any real numbers $a$ and $b$,

$$
E[a \xi+b \eta]=a E[\xi]+b E[\eta] .
$$

Remark 1: Uncertainty theory (Liu [10], Liu [14]) is a branch of mathematics for modeling belief degrees, while probability theory (Kolmogorov [9]) is for modeling frequencies. The main different is that the product uncertain measure is the minimum of uncertain measures of uncertain events, i.e.,

$$
\mathcal{M}\left\{\Lambda_{1} \times \Lambda_{2}\right\}=\mathcal{M}\left\{\Lambda_{1}\right\} \wedge \mathcal{M}\left\{\Lambda_{2}\right\},
$$

for uncertain events $\Lambda_{1}$ and $\Lambda_{2}$. The product probability measure is the product of probability measures of random events, i.e.,

$$
\operatorname{Pr}\left\{\Delta_{1} \times \Delta_{2}\right\}=\operatorname{Pr}\left\{\Delta_{1}\right\} \times \operatorname{Pr}\left\{\Delta_{2}\right\},
$$

for random events $\Delta_{1}$ and $\Delta_{2}$. It implies that uncertain variables and random variables obey different operational laws.

\section{PROBLEM FORMULATION}

Manpower planning consists of putting right number of employees, right kind of employees at the right place, right time, doing the right tasks for which they are suited for the achievement of different goals. The hierarchical system can be interpreted as a pyramid with lowest rank of job category at the base, for example, junior production worker. Moving up to higher levels, there are higher ranks of job categories, such as senior production worker, supervisor, senior supervisor, and manager. For each level, there will be personnel change through recruitment, promotion, dismissal, as well as employees quitting. The first three activities are regarded as decision variables because they can be decided by human resource department. But, the quitting rate is determined by employees' preference, which is beyond the control of human resource department.

In 2016, Sun et al. [16] constructed a stochastic discrete time optimization model for a manpower planning problem in stochastic environment, where the quitting rate is considered as a random variable. The objective is to minimize the expected human resource cost while maximizing the total expected production outputs. However, the quitting rate can be realistically regarded as a random variable only when the historical data of quitting rate is large enough. This is a fundamental premise. When the historical data is inadequate, it will be more appropriate to formulate it as an uncertain variable. In the following, we consider a manpower planning optimization problem for a manufacturing company with hierarchical system under uncertain environment. 
Let $M$ be the number of staff levels and $T$ be the number of time periods considered. The initial total number of staff in the company is $N$. To formulate the mathematical optimization model, we introduce the following notations:

$x_{i}(t)$ : the number of staff in level $i$ at the end of period $t$,

$R_{i}(t)$ : the recruitment number of staff being recruited to level $i$ at the end of period $t$,

$P_{i}(t)$ : the promotion number of staff being promoted to level $i$ at the end of period $t$,

$S_{i}(t)$ : the dismissal number of staff in level $i$ at the end of period $t$

$Q_{i}(t)$ : the quitting number of staff in level $i$ at the end of period $t$.

Here, $i$ represents the ith rank of job category.

For $i=1, \cdots, M, R_{i}(T)=0$ (there is no recruitment at the end of the planning horizon), $P_{i}(0)=S_{i}(0)=0$ (at the beginning of the time horizon the company only recruits, i.e., no promotion nor dismissal of staff) and $P_{M+1}(t)=0$ (no staff can be promoted into level $M+1$ at the end of period $t$ ), for $t=0, \cdots, T$. Here, we model the quitting rate $Q_{i}(t)$ as a normal uncertain variable with excepted value $\mu_{i}$ and variance $\sigma_{i}^{2}$. It is assumed that the quitting rates $Q_{i_{1}}\left(t_{1}\right)$ and $Q_{i_{2}}\left(t_{2}\right)$ are independent for any different periods $t_{1}$ and $t_{2}$ $\left(t_{1}, t_{2}=1, \cdots, T\right)$ or levels $i_{1}$ and $i_{2}\left(i_{1}, i_{2}=1, \cdots, M\right)$.

Because the quitting number $Q_{i}(t)(t=1, \cdots, T, i=$ $1, \cdots, M)$ is an uncertain variable, the number of staff in each hierarchy and period can be described by the following system of uncertain difference equations:

$$
\begin{aligned}
x_{i}(t+1)= & x_{i}(t)+R_{i}(t)+P_{i}(t)-P_{i+1}(t+1) \\
& -S_{i}(t+1)-Q_{i}(t+1), \\
& t=0, \cdots, T-1, i=1, \cdots, M .
\end{aligned}
$$

To maintain the company's normal operation, there should be enough of workers in each level. This situation is modeled by the following chance constraints:

$$
\mathcal{M}\left\{x_{i}(t) \geq p_{i}\right\} \geq q_{i}, \quad t=1, \cdots, T, i=1, \cdots, M,
$$

where $p_{i}$ is the pre-set minimum number of staff in level $i$ and $q_{i}$ is the given confidence level in level $i$ with $0<q_{i}<1$.

The objective of decision maker is to find an optimal strategy of recruitment, promotion and dismissal for minimizing the expected human resource cost while maximizing the total expected production outputs at the end of the planning horizon. Hence, $R_{i}(t), P_{i}(t)$, and $S_{i}(t)$ are decision variables, which are crisp in nature. Then the expected human resource cost and the expected total production outputs can be formulated as

$$
\begin{aligned}
g_{1}=E[ & \left.\sum_{t=0}^{T-1} \sum_{i=1}^{M} a_{i} x_{i}(t)\right]+\sum_{t=0}^{T-1} \sum_{i=1}^{M}\left\{b_{i}^{\text {ex }} R_{i}(t)+b_{i}^{\text {in }} P_{i}(t+1)\right. \\
& \left.+c_{i} S_{i}(t+1)\right\} \\
g_{2}=E[ & {\left[\sum_{t=0}^{T-1} \sum_{i=1}^{M} n_{i} x_{i}(t)\right] }
\end{aligned}
$$

respectively, where $a_{i}, b_{i}^{\text {ex }}, b_{i}^{\text {in }}$ and $c_{i}$ are the salary cost, recruitment and training cost of external workers, training cost of internal workers and dismissal cost per worker per period in level $i$, respectively, $n_{i}$ is the number of units that the workers in level $i$ can produce in each time period.

Obviously, this manpower planning optimization problem is a bi-objective optimization model. A common method for solving the bi-objective optimization model is to convert it into a single objective optimization model by introducing a weighting parameter, see, for example, Cai et al. [1], Deng et al. [7] and Sun et al. [16]. Likewise, we minimize the following function by introducing the weighting parameters $\nu$ and $1-\nu$ :

$$
\begin{aligned}
G_{0}=\nu g_{1}-(1-\nu) g_{2} \\
=\nu E\left[\sum_{t=0}^{T-1} \sum_{i=1}^{M} a_{i} x_{i}(t)\right]+\nu \sum_{t=0}^{T-1} \sum_{i=1}^{M}\left\{b_{i}^{\text {ex }} R_{i}(t)\right. \\
\left.\quad+b_{i}^{\text {in }} P_{i}(t+1)+c_{i} S_{i}(t+1)\right\} \\
\quad-(1-\nu) E\left[\sum_{t=0}^{T-1} \sum_{i=1}^{M} n_{i} x_{i}(t)\right] .
\end{aligned}
$$

Here, we use $-(1-\nu)$ because the original objective is to maximize the expected total production outputs function $g_{2}$. When $\nu=1$, the decision maker pays all its attention to minimize the expected human resource cost. Conversely, $\nu=0$ means that the decision maker just wants to maximize the expected production outputs. In this paper, we assume that $0<\nu<1$.

Then, we formulate the manpower planning problem under uncertain environment as the following uncertain discrete time optimization model, named as Problem $\mathbf{P}_{0}$ :

$$
\begin{gathered}
\min \quad G_{0}=\nu E\left[\sum_{t=0}^{T-1} \sum_{i=1}^{M} a_{i} x_{i}(t)\right]+\nu \sum_{t=0}^{T-1} \sum_{i=1}^{M}\left\{b_{i}^{\mathrm{ex}} R_{i}(t)\right. \\
\left.+b_{i}^{\text {in }} P_{i}(t+1)+c_{i} S_{i}(t+1)\right\} \\
-(1-\nu) E\left[\sum_{t=0}^{T-1} \sum_{i=1}^{M} n_{i} x_{i}(t)\right] \\
\text { s.t. } \quad x_{i}(t+1)=x_{i}(t)+R_{i}(t)+P_{i}(t)-P_{i+1}(t+1) \\
\quad-S_{i}(t+1)-Q_{i}(t+1), \\
\quad t=0, \cdots, T-1, i=1, \cdots, M, \\
\\
\quad x_{i}(0)=x_{i}^{0}, \quad i=1, \cdots, M, \\
\mathcal{M}\left\{x_{i}(t) \geq p_{i}\right\} \geq q_{i}, t=1, \cdots, T, i=1, \cdots, M \\
\quad R_{i}(t), P_{i}(t), S_{i}(t) \geq 0, t=0, \cdots, T, i=1, \cdots, M,
\end{gathered}
$$

where $0<\nu<1$.

Remark 2: Although the objective function in Sun et al. [16] contains the term of quitting cost, it has no influence on the transformation and optimization processes. Here, we assume that there is no withdrawal benefit for workers quitting the job themselves. We note that the summation operation at discrete time t means the human resource cost and production outputs during period $t+1$. Hence, the summation operation over $t$ from 0 to $T-1$ includes all the human resource cost and production outputs. 
Because $R_{i}(t), P_{i}(t)$, and $S_{i}(t)$ are decision variables, they are crisp rather than uncertain in nature. Then it follows from equations (5), (6) and Theorem 8 in Yang [19] that

$$
E\left[\sum_{t=0}^{T-1} \sum_{i=1}^{M} a_{i} x_{i}(t)\right]=\Delta-\sum_{t=0}^{T-1} \sum_{i=1}^{M} \sum_{j=0}^{t} a_{i} E\left[Q_{i}(j+1)\right],
$$

where the function $\Delta$ represents all crisp items. Thus, we can swap the order of the expected value operator $E$ and summation operator. The objective function $G_{0}$ can be rewritten as

$$
\begin{aligned}
G_{0}= & \nu \sum_{t=0}^{T-1} \sum_{i=1}^{M}\left\{a_{i} E\left[x_{i}(t)\right]+b_{i}^{\text {ex }} R_{i}(t)+b_{i}^{\text {in }} P_{i}(t+1)\right. \\
& \left.+c_{i} S_{i}(t+1)\right\}-(1-\nu) \sum_{t=0}^{T-1} \sum_{i=1}^{M} n_{i} E\left[x_{i}(t)\right] .
\end{aligned}
$$

\section{Deterministic TRANSFORMATION}

In this section, we first transform Problem $\mathbf{P}_{0}$ into an equivalent deterministic problem and then present a method for solving it.

Let $z_{i}(t)$ and $w_{i}(t)$ be new state variables defined by

$$
\begin{array}{r}
z_{i}(t)=E\left[x_{i}(t)\right], w_{i}(t)=\operatorname{Var}\left[x_{i}(t)\right], \quad t=0, \cdots, T, \\
i=1, \cdots, M .
\end{array}
$$

It follows from equations (5) and (6) that for $t=0, \cdots, T-1$ and $i=1, \cdots, M$,

$$
\begin{aligned}
z_{i}(t+1)= & E\left[x_{i}(t+1)\right] \\
= & E\left[x_{i}(t)+R_{i}(t)+P_{i}(t)-P_{i+1}(t+1)\right. \\
& \left.\quad-S_{i}(t+1)-Q_{i}(t+1)\right] \\
= & z_{i}(t)+R_{i}(t)+P_{i}(t)-P_{i+1}(t+1) \\
& -S_{i}(t+1)-\mu_{i} .
\end{aligned}
$$

Because the variables $x_{i}(t)$ and $Q_{i}(t+1)$ are essentially two independent normal uncertain variables, for $t=0, \cdots, T-1$ and $i=1, \cdots, M$, it follows from Theorem 5 in Yao [20] that

$$
\sqrt{w_{i}(t+1)}=\sqrt{\operatorname{Var}\left[x_{i}(t+1)\right]}=\sqrt{w_{i}(t)}+\sigma_{i} .
$$

We can find, in equation (16), the decision variables contain those in the past time $t$ as well as the present time $t+1$. For achieving the unity of time $t$, we define

$$
\begin{aligned}
v_{i}(t) & =P_{i}(t+1), t=0, \cdots, T-1, i=1, \cdots, M, \\
v_{M+i}(t) & =S_{i}(t+1), t=0, \cdots, T-1, i=1, \cdots, M .
\end{aligned}
$$

Then we transform $P_{i}(t)$ and $S_{i}(t)$ from decision variables into state variables with initial condition $P_{i}(0)=S_{i}(0)=0$ and introduce a new decision variable $v_{i}(t), t=0, \cdots, T-1, i=$ $1, \cdots, 2 M$. Hence, the decision variables are represented by $R_{i}(t), v_{i}(t), v_{M+i}(t)$ with $R_{i}(t), v_{i}(t), v_{M+i}(t) \geq 0, t=$ $0, \cdots, T-1, i=1, \cdots, M$.

The objective function (14) becomes

$$
\begin{gathered}
G_{0}=\sum_{t=0}^{T-1} \sum_{i=1}^{M}\left[\left(a_{i} \nu-(1-\nu) n_{i}\right) z_{i}(t)+b_{i}^{\text {ex }} R_{i}(t)\right. \\
\left.+b_{i}^{\text {in }} v_{i}(t)+c_{i} v_{M+i}(t)\right] .
\end{gathered}
$$

Theorem 1: The chance constraints (7) are equivalent to the following constraints.

$$
\begin{array}{r}
z_{i}(t)-p_{i}+\Phi^{-1}\left(1-q_{i}\right) \sqrt{w_{i}(t)} \geq 0, \quad t=1, \cdots, T, \\
i=1, \cdots, M,
\end{array}
$$

where $\Phi^{-1}$ is the inverse uncertainty distribution of normal uncertain variable with expected value 0 and variance 1 .

Proof Because $x_{i}(t)$ is a normal uncertain variable with expected value $z_{i}(t)$ and variance $w_{i}(t)$, we know that

$$
\frac{x_{i}(t)-z_{i}(t)}{\sqrt{w_{i}(t)}}, \quad t=1, \cdots, T, i=1, \cdots, M,
$$

is a normal uncertain variable with expected value 0 and variance 1 . Thus, the chance constraints (7) are equivalent to

$$
\mathcal{M}\left\{\frac{x_{i}(t)-z_{i}(t)}{\sqrt{w_{i}(t)}} \geq \frac{p_{i}-z_{i}(t)}{\sqrt{w_{i}(t)}}\right\} \geq q_{i},
$$

i.e.,

$$
\Phi^{-1}\left(1-q_{i}\right) \geq \frac{p_{i}(t)-z_{i}(t)}{\sqrt{w_{i}(t)}},
$$

where $\Phi^{-1}$ is the inverse uncertainty distribution of normal uncertain variable with expected value 0 and variance 1 . Thus, constraints (7) are equivalent to the constraints (21). The theorem is proved.

Because state variable $w_{i}(t)$ can be solved directly from equation (17) with initial value 0 , it can be obtained that

$$
w_{i}(t)=t^{2} \sigma_{i}^{2}, \quad t=0, \cdots, T, i=1, \cdots, M .
$$

Combining the state variables but excluding $w_{i}(t)$, for $t=$ $0, \cdots, T-1$, the new dynamical system can be written as

$$
\left(\begin{array}{c}
z_{1}(t+1) \\
\vdots \\
z_{M}(t+1) \\
P_{1}(t+1) \\
\vdots \\
P_{M}(t+1) \\
S_{1}(t+1) \\
\vdots \\
S_{M}(t+1)
\end{array}\right)=\left(\begin{array}{c}
z_{1}(t)+R_{1}(t)+P_{1}(t)-v_{2}(t) \\
-v_{M+1}(t)-\mu_{1} \\
\vdots \\
z_{M}(t)+R_{M}(t)+P_{M}(t)-0 \\
-v_{2 M}(t)-\mu_{M} \\
v_{1}(t) \\
\vdots \\
v_{M}(t) \\
v_{M+1}(t) \\
\vdots \\
v_{2 M}(t)
\end{array}\right),
$$

with initial condition $\left[x_{1}^{0}, \cdots, x_{M}^{0}, 0, \cdots, 0\right]^{\tau} \in \mathbb{R}^{3 M}$.

Based on the above operators, Problem $\mathbf{P}_{0}$ is equivalent to the following deterministic discrete time optimization model (24), named as Problem $\mathbf{P}_{1}$, where $0<\nu<1$.

\section{A. Solving Problem $\mathbf{P}_{1}$}

In this section, we convert Problem $\mathbf{P}_{1}$ into a standard linear programming problem such that it can be solved more easily.

The new state and decision variables can be rewritten in vector forms:

$$
\begin{aligned}
\boldsymbol{y}(t)= & {\left[z_{1}(t), \cdots, z_{M}(t), P_{1}(t), \cdots, P_{M}(t),\right.} \\
& \left.S_{1}(t), \cdots, S_{M}(t)\right]^{\tau}, \\
\boldsymbol{h}(t)= & {\left[R_{1}(t), \cdots, R_{M}(t), v_{1}(t), \cdots, v_{2 M}(t)\right]^{\tau} . }
\end{aligned}
$$




$$
\begin{aligned}
& \min G_{0}=\sum_{t=0}^{T-1} \sum_{i=1}^{M}\left[\left(a_{i} \nu-(1-\nu) n_{i}\right) z_{i}(t)+b_{i}^{\text {ex }} R_{i}(t)+b_{i}^{\text {in }} v_{i}(t)+c_{i} v_{M+i}(t)\right] \\
& \text { s.t. } \quad\left(\begin{array}{c}
z_{1}(t+1) \\
\vdots \\
z_{M}(t+1) \\
P_{1}(t+1) \\
\vdots \\
P_{M}(t+1) \\
S_{1}(t+1) \\
\vdots \\
S_{2 M}(t+1)
\end{array}\right)=\left(\begin{array}{c}
z_{1}(t)+R_{1}(t)+P_{1}(t)-v_{2}(t)-v_{M+1}(t)-\mu_{1} \\
\vdots \\
z_{M}(t)+R_{M}(t)+P_{M}(t)-0-v_{2 M}(t)-\mu_{M} \\
v_{1}(t) \\
\vdots \\
v_{M}(t) \\
v_{M+1}(t) \\
\vdots \\
v_{2 M}(t)
\end{array}\right), t=0, \cdots, T-1, \\
& {\left[z_{1}(0), \cdots, z_{M}(0), P_{1}(0), \cdots, P_{M}(0), S_{1}(0), \cdots, S_{M}(0)\right]^{\tau}=\left[x_{1}^{0}, \cdots, x_{M}^{0}, 0, \cdots, 0\right]^{\tau},} \\
& z_{i}(t)-p_{i}+\Phi^{-1}\left(1-q_{i}\right) \sqrt{w_{i}(t)} \geq 0, t=1, \cdots, T, i=1, \cdots, M, \\
& R_{i}(t), v_{i}(t), v_{M+i}(t) \geq 0, t=0, \cdots, T-1, i=1, \cdots, M .
\end{aligned}
$$

It follows from equations (27) and (28) that equation (24) can be rewritten as:

$$
G_{0}=\sum_{t=0}^{T-1}(\boldsymbol{\delta} \boldsymbol{y}(t)+\boldsymbol{\eta} \boldsymbol{h}(t))
$$

where $\boldsymbol{\delta}$ and $\boldsymbol{\eta}$ are both $3 M$ dimension row vectors given by

$$
\begin{aligned}
\boldsymbol{\delta}= & {\left[\left(a_{1} \nu-(1-\nu) n_{1}\right), \ldots,\left(a_{M} \nu-(1-\nu) n_{M}\right),\right.} \\
& 0, \ldots, 0], \\
\boldsymbol{\eta}= & {\left[b_{1}^{\mathrm{ex}}, \ldots, b_{M}^{\mathrm{ex}}, b_{1}^{\text {in }}, \ldots, b_{M}^{\text {in }}, c_{1}, \ldots, c_{M}\right] . }
\end{aligned}
$$

Let $\boldsymbol{I}$ be an $M \times M$ identity matrix, $\mathbf{0}$ be an $M \times M$ zero matrix, $\Lambda$ be an $M \times M$ matrix defined by

$$
\boldsymbol{\Lambda}=\left(\begin{array}{ccccc}
0 & 1 & 0 & \cdots & 0 \\
0 & 0 & 1 & \cdots & 0 \\
\vdots & \vdots & \vdots & \ddots & \vdots \\
0 & 0 & 0 & \cdots & 1 \\
0 & 0 & 0 & \cdots & 0
\end{array}\right)
$$

and

$$
\boldsymbol{\alpha}=\left(\begin{array}{lll}
\boldsymbol{I} & \boldsymbol{I} & \mathbf{0} \\
\mathbf{0} & \mathbf{0} & \mathbf{0} \\
\mathbf{0} & \mathbf{0} & \mathbf{0}
\end{array}\right), \boldsymbol{\beta}=\left(\begin{array}{ccc}
\boldsymbol{I} & -\boldsymbol{\Lambda} & -\boldsymbol{I} \\
\mathbf{0} & \boldsymbol{I} & \mathbf{0} \\
\mathbf{0} & \mathbf{0} & \boldsymbol{I}
\end{array}\right) .
$$

Theorem 2: For $t=1, \cdots, T$, the state variable $\boldsymbol{y}(t)$ can be expressed in terms of decision variable $\boldsymbol{h}(t)$ as given below:

$$
\boldsymbol{y}(t)=\boldsymbol{\alpha}^{t} \boldsymbol{y}^{0}+\sum_{k=0}^{t-1}\left(\boldsymbol{\alpha}^{(t-1)-k} \boldsymbol{\beta} \boldsymbol{h}(k)+\boldsymbol{\alpha}^{k} \boldsymbol{\zeta}\right),
$$

where $\boldsymbol{y}(0)=\boldsymbol{y}^{0}=\left[x_{1}^{0}, \cdots, x_{M}^{0}, 0, \cdots, 0\right]^{\tau} \in \mathbb{R}^{3 M}$ and $\boldsymbol{\zeta}$ is a $3 M$ dimensional column vector given by

$$
\boldsymbol{\zeta}=\left[-\mu_{1}, \cdots,-\mu_{M}, 0, \cdots, 0\right]^{\tau} .
$$

Proof Here, we use the induction method. It is clear that

$$
\boldsymbol{y}(1)=\boldsymbol{\alpha} \boldsymbol{y}^{0}+\boldsymbol{\beta} \boldsymbol{h}(0)+\boldsymbol{\zeta} .
$$

Suppose that equation (32) is satisfied when $t=n$. Then, at $t=n+1$, we have

$$
\begin{aligned}
\boldsymbol{y}(n+1)= & \boldsymbol{\alpha} \boldsymbol{y}(n)+\boldsymbol{\beta} \boldsymbol{h}(n)+\boldsymbol{\zeta} \\
= & \boldsymbol{\alpha}\left[\boldsymbol{\alpha}^{n} \boldsymbol{y}^{0}+\sum_{k=0}^{n-1}\left(\boldsymbol{\alpha}^{(n-1)-k} \boldsymbol{\beta} \boldsymbol{h}(k)+\boldsymbol{\alpha}^{k} \boldsymbol{\zeta}\right)\right] \\
& +\boldsymbol{\beta} \boldsymbol{h}(n)+\boldsymbol{\zeta} \\
= & \boldsymbol{\alpha}^{n+1} \boldsymbol{y}^{0}+\sum_{k=0}^{n}\left(\boldsymbol{\alpha}^{n-k} \boldsymbol{\beta} \boldsymbol{h}(k)+\boldsymbol{\alpha}^{k} \boldsymbol{\zeta}\right) .
\end{aligned}
$$

The theorem is proved.

Also, the state constraints (25) can be written in a matrix form given by

$$
\boldsymbol{\kappa} \boldsymbol{y}(t) \geq \boldsymbol{\lambda}(t), \quad t=1, \cdots, T,
$$

where $\boldsymbol{\kappa}$ is an $M \times 3 M$ matrix, and $\boldsymbol{\lambda}(t)$ is an $M$ dimensional column vector defined by

$$
\kappa=\left(\begin{array}{lll}
\boldsymbol{I} & \mathbf{0} & \mathbf{0}
\end{array}\right)
$$

and

$$
\boldsymbol{\lambda}(t)=\left(\begin{array}{c}
p_{1}-\Phi^{-1}\left(1-q_{1}\right) \sqrt{w_{1}(t)} \\
\vdots \\
p_{M}-\Phi^{-1}\left(1-q_{M}\right) \sqrt{w_{M}(t)}
\end{array}\right) .
$$

For translating the inequality constraints (34) into equality constraints, we add an auxiliary decision variable to the left hand side of (34), yielding

$$
\boldsymbol{\kappa} \boldsymbol{y}(t)-\boldsymbol{\theta}(t)=\boldsymbol{\lambda}(t), \quad t=1, \cdots, T,
$$

where $\boldsymbol{\theta}(t)=\left[\theta_{1}(t), \cdots, \theta_{M}(t)\right]^{\tau} \in \mathbb{R}^{M}$ with $\theta_{i}(t) \geq 0$, $i=1, \cdots, M$.

Define

$$
\boldsymbol{\omega}=\left[\boldsymbol{h}(0)^{\tau}, \cdots, \boldsymbol{h}(T-1)^{\tau}, \boldsymbol{\theta}(1)^{\tau}, \cdots, \boldsymbol{\theta}(T)^{\tau}\right]^{\tau} \in \mathbb{R}^{4 M T}
$$


Obviously, we have

$$
\omega \geq \mathbf{0},
$$

where $\mathbf{0}$ is a $4 M T$ dimensional column vector.

According to equations (29), (32) and (38), the objective function $G_{0}$ can be expressed as a linear function:

$$
G_{0}=c^{\tau} \omega
$$

where $c$ is a $4 M T$ dimensional column vector defined by

$$
\begin{aligned}
\boldsymbol{c}= & {\left[\sum_{t=0}^{T-2} \boldsymbol{\delta} \boldsymbol{\alpha}^{t} \boldsymbol{\beta}+\boldsymbol{\eta}, \sum_{t=0}^{T-3} \boldsymbol{\delta} \boldsymbol{\alpha}^{t} \boldsymbol{\beta}+\boldsymbol{\eta}, \cdots, \boldsymbol{\delta} \boldsymbol{\beta}+\boldsymbol{\delta} \boldsymbol{\alpha} \boldsymbol{\beta}+\boldsymbol{\eta},\right.} \\
& \boldsymbol{\delta} \boldsymbol{\beta}+\boldsymbol{\eta}, \boldsymbol{\eta}, \mathbf{0}]^{\tau} .
\end{aligned}
$$

Substituting equation (32) into equation (37), the constraints (37) can be expressed as

$$
A \omega=b,
$$

where $\boldsymbol{A}$ and $\boldsymbol{b}$ are given below as an $M T \times 4 M T$ matrix

$$
\left(\begin{array}{cccccccc}
\kappa \boldsymbol{\beta} & \overline{\mathbf{0}} & \cdots & \overline{\mathbf{0}} & -\boldsymbol{I} & \mathbf{0} & \cdots & \mathbf{0} \\
\kappa \boldsymbol{\alpha} \boldsymbol{\beta} & \boldsymbol{\kappa} \boldsymbol{\beta} & \cdots & \overline{\mathbf{0}} & \mathbf{0} & -\boldsymbol{I} & \cdots & \mathbf{0} \\
\vdots & \vdots & \ddots & \vdots & \vdots & \vdots & \ddots & \vdots \\
\kappa \boldsymbol{\alpha}^{T-1} \boldsymbol{\beta} & \kappa \boldsymbol{\alpha}^{T-2} \boldsymbol{\beta} & \cdots & \boldsymbol{\kappa} \boldsymbol{\beta} & \mathbf{0} & \mathbf{0} & \cdots & -\boldsymbol{I}
\end{array}\right)
$$

and an $M T$ column vector

$$
\left(\begin{array}{c}
\lambda(1)-\kappa \boldsymbol{\alpha} \boldsymbol{y}^{0}-\kappa \boldsymbol{\zeta} \\
\boldsymbol{\lambda}(2)-\kappa \boldsymbol{\alpha}^{2} \boldsymbol{y}^{0}-\kappa \boldsymbol{\zeta}-\boldsymbol{\alpha} \boldsymbol{\zeta} \\
\vdots \\
\boldsymbol{\lambda}(T)-\boldsymbol{\kappa} \boldsymbol{\alpha}^{T} \boldsymbol{y}^{0}-\sum_{t=0}^{T-1} \boldsymbol{\kappa} \boldsymbol{\alpha}^{t} \boldsymbol{\zeta}
\end{array}\right),
$$

respectively. Here, $\overline{\mathbf{0}}$ is an $M \times 3 M$ zero matrix.

Based on equations (39), (40) and (42), Problem $\mathbf{P}_{1}$ can be formally stated as a standard linear programming problem as given in the following:

$$
\begin{array}{cl}
\min & \boldsymbol{c}^{\tau} \boldsymbol{\omega} \\
\text { s.t. } & \boldsymbol{A} \boldsymbol{\omega}=\boldsymbol{b}, \\
& \boldsymbol{\omega} \geq \mathbf{0} .
\end{array}
$$

Then, we use the interior-point linear programming solver SDPT3 (MATLAB platform) proposed in Toh et al. [17] to solve the converted standard linear programming problem (45). After obtaining the value of $\omega^{*}$, the procedures for obtaining the solution of the original Problem $\mathbf{P}_{0}$ are summarized as follows:

Step 1: Calculate the values of $\boldsymbol{h}(0), \cdots, \boldsymbol{h}(T-1)$ by equation (38);

Step 2: Calculate the values of decision variables of the original Problem $\mathbf{P}_{0}$ according to equations (18), (19) and (28).

\section{NUMERICAL SIMUlation}

In this section, we use the same data and parameters, shown in Tables I and II, as in Sun et al. [16] to carry out a numerical simulation and make a comparison. All values in Table II are in US dollars. Also, we choose initial workforce $N=1000$, planning horizon $T=12$ months, confidence level $q_{i}=0.8$ $(i=1,2, \cdots, 6)$ and weighting parameter $\nu=0.3$. Because the quitting cost per worker is chosen as 0 in Sun et al. [16], the optimization problem can be modeled as defined in Section III.

Here, we use the interior-point linear programming solver SDPT3 (MATLAB platform) proposed in Toh et al. [17] to solve the converted standard linear programming problem. From the calculation procedure presented in Section IV, the optimal manpower strategies of recruitment, promotion and dismissal for three different countries can be calculated. The obtained solutions are given in Tables III, V and VII. Our mathematical model for the uncertain manpower planning optimization problem provides results that realistically reflect the conditions of each of the countries considered. We choose three countries (Singapore, Denmark and China) that are distinct in nature and especially in salaries. One is a developed country, Denmark, which has relatively stable domestic labor with relatively high wages. At the other extreme is China, a developing country with relatively low wages and plenty of available manpower. The third is Singapore. While a developed country, Singapore utilizes a huge number of cheap unskilled foreign labor to keep manpower costs down at the lower levels. The results of our model demonstrate that the inclusion of the effect of production outputs produces results that are realistic and takes into consideration the differences of each country.

Singapore, with relatively cheap and abundant low-skilled foreign labor, results in an optimal workforce that encourages recruitment at the lowest two levels as shown in Table III, and also at the more expensive and skilled managerial levels. It is cheaper to recruit than promote internal skilled staff at the managerial levels. Denmark, having the most stable workforce with relatively higher wages than the other two countries, achieves workforce stability in a much shorter time than Singapore and China. From Table V, their human resource strategy stabilizes after nine months while there are no signs of workforce stability in Singapore and China. With regards to recruitment and promotion, the strategy suitable for Denmark is to recruit at all levels rather than promote internal staff as the promotion cost is higher than the recruitment cost for the country. China has a huge number of cheap domestic workers. Hence, it is hardly surprising that their optimal workforce recommends recruiting a huge, even excessive, number of workers at all levels as in Table VII. Their model does not recommend recruitment or promotion after the initial recruitment.

The optimal manpower structures in each level for three different countries can be found in Tables IV, VI and VIII, which are demonstrated in Figures 1, 2 and 3, respectively. From Tables IV, VI and VIII or Figures 1, 2 and 3, there are more staff at the end of each period for China than Singapore and Denmark. This is reasonable because China has abundant and cheap domestic workforce. The optimal manpower cost and units of output are $\$ 21,339,468.64$ and 957,150 for Singapore, $\$ 59,635,567.72$ and 952,830 for Denmark, $\$ 5,778,962.99$ and $1,421,890$ for China.

Modeling the quitting rate to better reflect reality results in more employees in the optimal manpower structure. While more employees result in higher manpower cost across all countries, it also leads to higher number of produced outputs. 
TABLE IX

The Unit Cost of Two Different Models

\begin{tabular}{ccc}
\hline Country & Sun et al. [16] model & Our model \\
\hline Singapore & 22.3476 & 22.2948 \\
Denmark & 62.4880 & 62.5878 \\
China & 4.1055 & 4.0643 \\
\hline
\end{tabular}

Comparing our results with Sun et al. [16], the percentage increase in manpower cost is $2.2302 \%, 2.1731 \%$ and $3.4213 \%$ for Singapore, Denmark and China, respectively. The corresponding increase in production output as a result of the manpower increase is $2.4728 \%, 2.0103 \%$ and $4.4715 \%$. Table IX illustrates the unit output cost based on our optimal manpower structure compared to the optimization model in Sun et al. [16].

Our model, with more stable retention of employees, sees a lower unit cost for Singapore and China, but a higher unit output cost for Denmark. This, indeed, reflects more accurately the real situation, as the manpower cost in Singapore is approximately four times that of China, while in Denmark, the manpower cost is approximately ten times that of China. Thus, unless the quality of the product produced in Denmark is substantially higher than that of Singapore and China, the manufacturing industry is unable to compete with that of Singapore and China.

Our model confirms that for labour intensive companies producing low value products, it is more profitable to base their operations in low labour cost countries like China or countries where the labour cost is relatively lower such as Singapore for which a large number of low cost foreign workers is used. In conclusion, for high labour cost countries like Denmark, only high end products will be compatible and survive.

\section{CONCLUSION}

In this paper, we discussed a manpower planning optimization problem for a hierarchical system under uncertain environment because the quitting rate of employees is usually uncertain in nature. The manpower planning optimization problem was formulated as an uncertain discrete time optimization model for minimising the expected human resource cost while maximizing the expected production outputs. Our model considered the actual personnel change through recruitment, promotion, dismissal, as well as employees quitting and took into account the influence of the production outputs of workers. Then, we transformed it into an equivalent deterministic problem and presented a solution method for solving it. In order to show the effectiveness and rationality of our model, we remodeled the practical example considered in Sun et al. [16] by our method. The obtained results showed that our model reflects more accurately the real situation than the stochastic model.

\section{ACKNOWLEDGMENT}

This work is supported by the National Natural Science Foundation of China (No.61673011).

\section{REFERENCES}

[1] X. Cai, K. L. Teo, X. Yang, and X. Zhou, "Portfolio optimization under a minimax rule," Manag. Sci., vol. 46, no. 7, pp. 957-972, 2000.

[2] A. Chattopadhyay and A. Gupta, "A stochastic manpower planning model under varying class sizes," Ann. Oper. Res., vol. 155, no. 1, pp. 41-49, 2007.

[3] X. Chen, "Variation analysis of uncertain stationary independent increment processes," Eur. J. Oper. Res., vol. 222, no. 2, pp. 312-316, 2012.

[4] X. Chen and J. Gao, "Uncertain term structure model of interest rate," Soft Comput., vol. 17, no. 4, pp. 597-604, 2013.

[5] X. Chen and B. Liu, "Existence and uniqueness theorem for uncertain differential equations," Fuzzy Optim. Dec. Making, vol. 2, no. 9, pp. 69$81,2010$.

[6] X. Chen, Y. Liu, and D. Ralescu, "Uncertain stock model with periodic dividends," Fuzzy Optim. Dec. Making, vol. 12, no. 1, pp. 111-123, 2013.

[7] X. Deng, Z. Li, and S. Wang, "A minimax portfolio selection strategy with equilibrium," Eur. J. Oper. Res., vol. 166, no. 1, pp. 278-292, 2005.

[8] Y. Gao, "Uncertain models for single facility location problems on networks," Appl. Math. Model., vol. 36, no. 6, pp. 2592-2599, 2012.

[9] A. Kolmogorov, Grundbegriffe der Wahrscheinlichkeitsrechnung, Berlin, Germany: Julius-Springer, 1933.

[10] B. Liu, Uncertainty Theory, 2nd ed., Berlin, Germany: Springer-Verlag, 2007.

[11] B. Liu, "Fuzzy process, hybrid process and uncertain process," $J$. Uncertain Syst., vol. 2, no. 1, pp. 3-16, 2008.

[12] B. Liu, "Some research problems in uncertainty theory," J. Uncertain Syst., vol. 3, no. 1, pp. 3-10, 2009.

[13] B. Liu, Theory and Practice of Uncertain Programming, 2nd ed., Berlin, Germany: Springer-Verlag, 2009.

[14] B. Liu, Uncertainty Theory: A Branch of Mathematics for Modeling Human Uncertainty, Berlin, Germany: Springer-Verlag, 2010.

[15] Y. Sheng and S. Kar, "Some results of moments of uncertain variable through inverse uncertainty distribution," Fuzzy Optim. Dec. Making, vol. 14, no. 1, pp. 57-76, 2015.

[16] Y. Sun, G. Aw, C. Yu, K. L. Teo, and X. Chen, "Multi-period stochastic manpower and produvtivity optimization in a hierarchiacl system," Pac. J. Optim., vol. 12, no. 3, pp. 521-539, 2016.

[17] K. Toh, M. Todd, and R. Tütüncü, "On the implementation and usage of SDPT3-a Matlab software package for semidefinite-quadratic-linear programming, version 4.0," Int. Ser. Oper. Res. Manag. Sci., vol. 166, pp. 715-754, 2012.

[18] S. Yan, C. Chen, and M. Chen, "Stochastic models for air cargo terminal manpower supply planning in long term operations," Appl. Stoch. Model. Bus., vol. 24, no. 3, pp. 261-275, 2008.

[19] X. Yang, "On comonotonic functions of uncertain variables," Fuzzy Optim. Dec. Making, vol. 12, no. 1, pp. 89-98, 2013.

[20] K. Yao, "A formula to calculate the variance of uncertain variable," Soft Comput., vol. 19, no. 10, pp. 2947-2953, 2015.

[21] K. Yao, J. Gao, and Y. Gao, "Some stability theorems of uncertain differential equation," Fuzzy Optim. Dec. Making, vol. 12, no. 1, pp. 313, 2013.

[22] K. Yao and X. Li, "Uncertain alternating renewal process and its application," IEEE Trans. Fuzzy Syst., vol. 20, no. 6, pp. 1154-1160, 2012.

[23] A. Young and P. Vassiliou, "A non-linear model on the promotion of staff," J. Roy. Stat. Soc. A, vol. 173, no. 4, pp. 584-595, 1974.

[24] Y. Zhu, "Uncertain optimal control with application to a portfolio selection model," Cybernet Syst., vol. 41, no. 7, pp. 535-547, 2010.

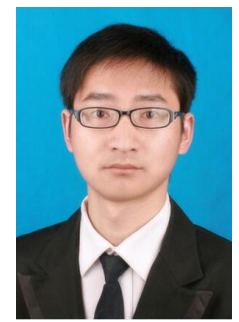

Bo Li received the Ph.D. degree from Nanjing University of Science and Technology, Nanjing, China, in 2017.

$\mathrm{He}$ is currently a lecturer with the School of Applied Mathematics, Nanjing University of Finance and Economics, Nanjing, China. His research interests include optimal control, uncertain differential equation and uncertain portfolio selection. 
TABLE I

Initial Hierarchy Structure, Minimum Staff number, Productivity, Mean and Variance of Quitting Rate

\begin{tabular}{ccccccc}
\hline & Level 1 & Level 2 & Level 3 & Level 4 & Level 5 & Level 6 \\
\hline$x_{i}^{0}$ & 350 & 250 & 150 & 120 & 80 & 50 \\
$p_{i}$ & 250 & 170 & 100 & 75 & 50 & 25 \\
$n_{i}$ & 100 & 120 & 150 & 40 & 60 & 90 \\
$\mu_{i}$ & 30 & 20 & 10 & 5 & 3 & 1 \\
$\sigma_{i}^{2}$ & 9 & 6.25 & 4 & 0.5625 & 0.25 & 0.01 \\
\hline
\end{tabular}

TABLE II

VARIOUS MANPOWER COST OF EACH HIERARChy LEVEL

\begin{tabular}{ccccccc}
\hline Singapore & Level 1 & Level 2 & Level 3 & Level 4 & Level 5 & Level 6 \\
\hline$a_{i}$ & 800.00 & 960.00 & 1728.00 & 3110.40 & 5598.72 & 11197.44 \\
$b_{i}^{\text {ex }}$ & 2000.00 & 2400.00 & 4320.00 & 6842.88 & 12317.18 & 19035.65 \\
$b_{i}^{\text {nn }}$ & 2000.00 & 1056.00 & 1900.80 & 3421.44 & 6158.59 & 12317.18 \\
$c_{i}$ & 800.00 & 960.00 & 1728.00 & 3110.40 & 5598.72 & 11197.44 \\
\hline Denmark & Level 1 & Level 2 & Level 3 & Level 4 & Level 5 & Level 6 \\
\hline$a_{i}$ & 3206.67 & 4329.00 & 5844.15 & 8181.81 & 11454.53 & 16036.35 \\
$b_{i}^{\text {ex }}$ & 8016.67 & 10822.50 & 14610.38 & 17999.98 & 25199.97 & 27261.79 \\
$b_{i}^{\text {in }}$ & 8016.67 & 4761.90 & 6428.57 & 8999.99 & 12599.99 & 17639.98 \\
$c_{i}$ & 3206.67 & 4329.00 & 5844.15 & 8181.81 & 11454.53 & 16036.35 \\
\hline China & Level 1 & Level 2 & Level 3 & Level 4 & Level 5 & Level 6 \\
\hline$a_{i}$ & 206.00 & 247.20 & 321.36 & 514.18 & 1079.77 & 3239.31 \\
$b_{i}^{\text {ex }}$ & 515.00 & 618.00 & 803.40 & 1131.19 & 2375.49 & 5506.82 \\
$b_{i}^{\text {nn }}$ & 515.00 & 271.92 & 353.50 & 565.59 & 1187.75 & 3563.24 \\
$c_{i}$ & 206.00 & 247.20 & 321.36 & 514.18 & 1079.77 & 3239.31 \\
\hline & & & & & &
\end{tabular}

TABLE III

OPTIMAL HR STRATEGY OF SiNGAPORE

\begin{tabular}{|c|c|c|c|c|c|c|}
\hline Recruitment & Level 1 & Level 2 & Level 3 & Level 4 & Level 5 & Level 6 \\
\hline$t=0$ & 0 & 0 & 0 & 0 & 0 & 0 \\
\hline$t=1$ & 0 & 0 & 0 & 0 & 0 & 0 \\
\hline$t=2$ & 0 & 0 & 0 & 0 & 0 & 0 \\
\hline$t=3$ & 29 & 15 & 0 & 0 & 0 & 0 \\
\hline$t=4$ & 32 & 33 & 0 & 0 & 0 & 0 \\
\hline $\mathrm{t}=5$ & 32 & 33 & 0 & 0 & 0 & 0 \\
\hline$t=6$ & 32 & 33 & 0 & 0 & 0 & 0 \\
\hline$t=7$ & 32 & 33 & 0 & 0 & 0 & 0 \\
\hline$t=8$ & 32 & 33 & 0 & 5 & 0 & 0 \\
\hline $\mathrm{t}=9$ & 32 & 33 & 0 & 5 & 3 & 0 \\
\hline$t=10$ & 32 & 33 & 0 & 5 & 3 & 1 \\
\hline$t=11$ & 32 & 21 & 0 & 5 & 3 & 1 \\
\hline$t=12$ & 0 & 0 & 0 & 0 & 0 & 0 \\
\hline Promotion & Level 1 & Level 2 & Level 3 & Level 4 & Level 5 & Level 6 \\
\hline$t=0$ & 0 & 0 & 0 & 0 & 0 & 0 \\
\hline$t=1$ & 0 & 0 & 0 & 0 & 0 & 0 \\
\hline$t=2$ & 0 & 0 & 0 & 0 & 0 & 0 \\
\hline$t=3$ & 0 & 0 & 0 & 0 & 0 & 0 \\
\hline $\mathrm{t}=4$ & 0 & 0 & 7 & 0 & 0 & 0 \\
\hline$t=5$ & 0 & 0 & 11 & 0 & 0 & 0 \\
\hline$t=6$ & 0 & 0 & 11 & 0 & 0 & 0 \\
\hline$t=7$ & 0 & 0 & 11 & 0 & 0 & 0 \\
\hline$t=8$ & 0 & 0 & 11 & 0 & 0 & 0 \\
\hline $\mathrm{t}=9$ & 0 & 0 & 11 & 0 & 0 & 0 \\
\hline$t=10$ & 0 & 0 & 11 & 0 & 0 & 0 \\
\hline$t=11$ & 0 & 0 & 11 & 0 & 0 & 0 \\
\hline$t=12$ & 0 & 0 & 0 & 0 & 0 & 0 \\
\hline Dismissal & Level 1 & Level 2 & Level 3 & Level 4 & Level 5 & Level 6 \\
\hline$t=0$ & 0 & 0 & 0 & 0 & 0 & 0 \\
\hline$t=1$ & 0 & 0 & 0 & 0 & 0 & 14 \\
\hline$t=2$ & 0 & 0 & 0 & 0 & 0 & 0 \\
\hline$t=3$ & 0 & 0 & 0 & 0 & 0 & 0 \\
\hline $\mathrm{t}=4$ & 0 & 0 & 0 & 0 & 0 & 0 \\
\hline $\mathrm{t}=5$ & 0 & 0 & 0 & 0 & 0 & 0 \\
\hline $\mathrm{t}=6$ & 0 & 0 & 0 & 0 & 0 & 0 \\
\hline $\mathrm{t}=7$ & 0 & 0 & 0 & 0 & 0 & 0 \\
\hline $\mathrm{t}=8$ & 0 & 0 & 0 & 0 & 0 & 0 \\
\hline $\mathrm{t}=9$ & 0 & 0 & 0 & 0 & 0 & 0 \\
\hline $\mathrm{t}=10$ & 0 & 0 & 0 & 0 & 0 & 0 \\
\hline $\mathrm{t}=11$ & 0 & 0 & 0 & 0 & 0 & 0 \\
\hline $\mathrm{t}=12$ & 0 & 0 & 0 & 0 & 0 & 0 \\
\hline
\end{tabular}


TABLE IV

Optimal Manpower Structure of Singapore

\begin{tabular}{cccccccc}
\hline No. of workers & Level 1 & Level 2 & Level 3 & Level 4 & Level 5 & Level 6 & Total \\
\hline $\mathrm{t}=0$ & 350 & 250 & 150 & 120 & 80 & 50 & 1000 \\
$\mathrm{t}=1$ & 320 & 230 & 140 & 115 & 77 & 35 & 917 \\
$\mathrm{t}=2$ & 290 & 210 & 130 & 110 & 74 & 34 & 848 \\
$\mathrm{t}=3$ & 260 & 190 & 120 & 105 & 71 & 33 & 779 \\
$\mathrm{t}=4$ & 259 & 178 & 110 & 100 & 68 & 32 & 747 \\
$\mathrm{t}=5$ & 261 & 180 & 107 & 95 & 65 & 31 & 739 \\
$\mathrm{t}=6$ & 263 & 182 & 108 & 90 & 62 & 30 & 735 \\
$\mathrm{t}=7$ & 265 & 184 & 109 & 85 & 59 & 29 & 731 \\
$\mathrm{t}=8$ & 267 & 186 & 110 & 80 & 56 & 28 & 727 \\
$\mathrm{t}=9$ & 269 & 188 & 111 & 80 & 53 & 27 & 728 \\
$\mathrm{t}=10$ & 271 & 190 & 112 & 80 & 53 & 26 & 732 \\
$\mathrm{t}=11$ & 273 & 192 & 113 & 80 & 53 & 26 & 737 \\
$\mathrm{t}=12$ & 275 & 193 & 114 & 80 & 53 & 26 & 741 \\
\hline
\end{tabular}

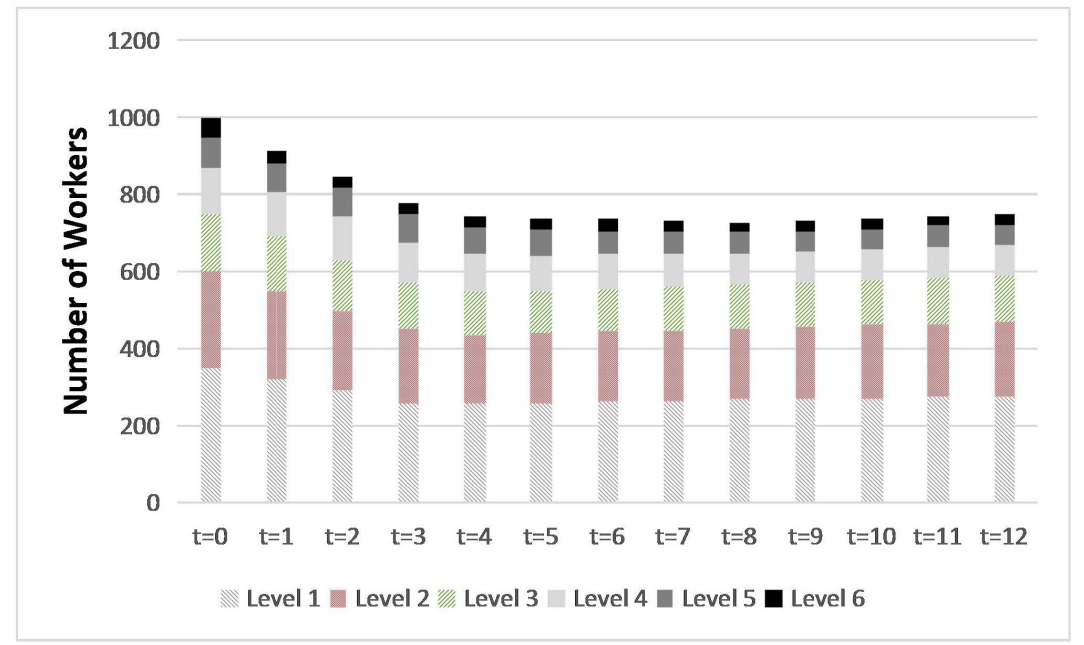

Fig. 1. Optimal Manpower Structure of Singapore

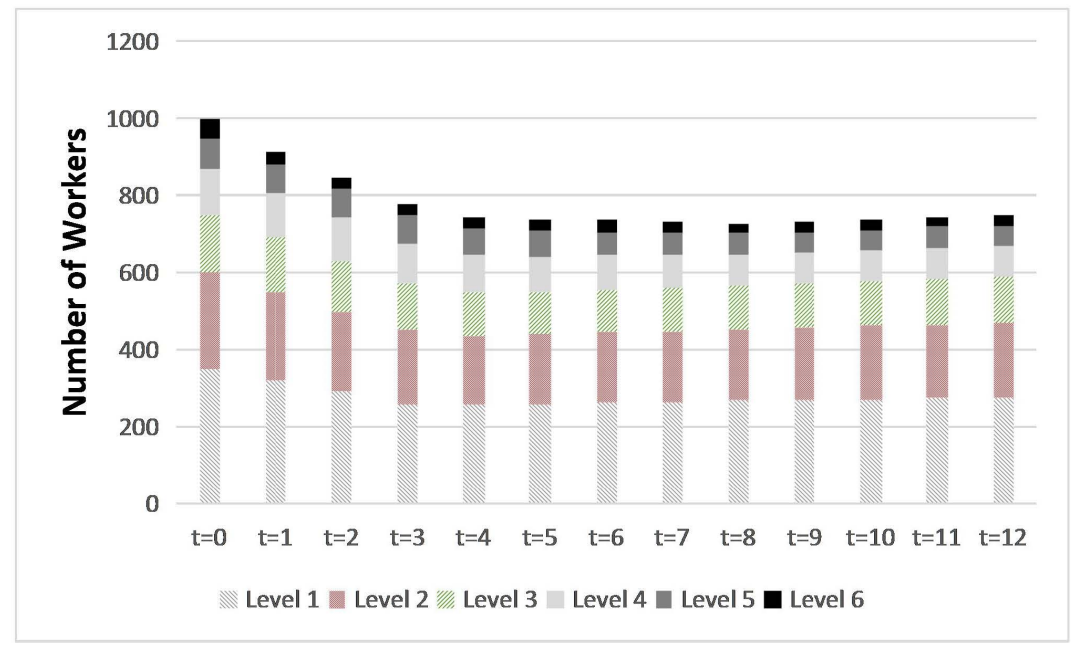

Fig. 2. Optimal Manpower Structure of Denmark

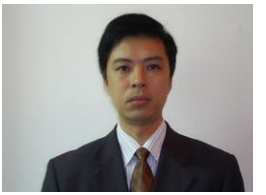

Yuanguo Zhu received the Ph.D. degree from Tsinghua University, Beijing, China, in 2004.

$\mathrm{He}$ is currently a professor with the School of Science, Nanjing University of Science and Technology, Nanjing, China. His research interests include optimal control, optimization and intelligent computing. 
TABLE V

OPTIMAL HR STRATEGY OF DENMARK

\begin{tabular}{|c|c|c|c|c|c|c|}
\hline Recruitment & Level 1 & Level 2 & Level 3 & Level 4 & Level 5 & Level 6 \\
\hline$t=0$ & 0 & 0 & 0 & 0 & 0 & 0 \\
\hline $\mathrm{t}=1$ & 0 & 0 & 0 & 0 & 0 & 0 \\
\hline$t=2$ & 0 & 0 & 0 & 0 & 0 & 0 \\
\hline$t=3$ & 29 & 7 & 0 & 0 & 0 & 0 \\
\hline$t=4$ & 32 & 21 & 7 & 0 & 0 & 0 \\
\hline$t=5$ & 32 & 21 & 11 & 0 & 0 & 0 \\
\hline$t=6$ & 32 & 21 & 11 & 0 & 0 & 0 \\
\hline$t=7$ & 32 & 21 & 11 & 0 & 0 & 0 \\
\hline$t=8$ & 32 & 21 & 11 & 5 & 0 & 0 \\
\hline$t=9$ & 32 & 21 & 11 & 5 & 3 & 0 \\
\hline$t=10$ & 32 & 21 & 11 & 5 & 3 & 1 \\
\hline$t=11$ & 32 & 21 & 11 & 5 & 3 & 1 \\
\hline$t=12$ & 0 & 0 & 0 & 0 & 0 & 0 \\
\hline Promotion & Level 1 & Level 2 & Level 3 & Level 4 & Level 5 & Level 6 \\
\hline$t=0$ & 0 & 0 & 0 & 0 & 0 & 0 \\
\hline $\mathrm{t}=1$ & 0 & 0 & 0 & 0 & 0 & 0 \\
\hline$t=2$ & 0 & 0 & 0 & 0 & 0 & 0 \\
\hline$t=3$ & 0 & 0 & 0 & 0 & 0 & 0 \\
\hline$t=4$ & 0 & 0 & 0 & 0 & 0 & 0 \\
\hline$t=5$ & 0 & 0 & 0 & 0 & 0 & 0 \\
\hline$t=6$ & 0 & 0 & 0 & 0 & 0 & 0 \\
\hline$t=7$ & 0 & 0 & 0 & 0 & 0 & 0 \\
\hline$t=8$ & 0 & 0 & 0 & 0 & 0 & 0 \\
\hline$t=9$ & 0 & 0 & 0 & 0 & 0 & 0 \\
\hline$t=10$ & 0 & 0 & 0 & 0 & 0 & 0 \\
\hline$t=11$ & 0 & 0 & 0 & 0 & 0 & 0 \\
\hline$t=12$ & 0 & 0 & 0 & 0 & 0 & 0 \\
\hline Dismissal & Level 1 & Level 2 & Level 3 & Level 4 & Level 5 & Level 6 \\
\hline$t=0$ & 0 & 0 & 0 & 0 & 0 & 0 \\
\hline$t=1$ & 0 & 0 & 0 & 0 & 0 & 14 \\
\hline$t=2$ & 0 & 0 & 0 & 0 & 0 & 0 \\
\hline$t=3$ & 0 & 0 & 0 & 0 & 0 & 0 \\
\hline$t=4$ & 0 & 0 & 0 & 0 & 0 & 0 \\
\hline$t=5$ & 0 & 0 & 0 & 0 & 0 & 0 \\
\hline$t=6$ & 0 & 0 & 0 & 0 & 0 & 0 \\
\hline$t=7$ & 0 & 0 & 0 & 0 & 0 & 0 \\
\hline$t=8$ & 0 & 0 & 0 & 0 & 0 & 0 \\
\hline$t=9$ & 0 & 0 & 0 & 0 & 0 & 0 \\
\hline$t=10$ & 0 & 0 & 0 & 0 & 0 & 0 \\
\hline$t=11$ & 0 & 0 & 0 & 0 & 0 & 0 \\
\hline$t=12$ & 0 & 0 & 0 & 0 & 0 & 0 \\
\hline
\end{tabular}

TABLE VI

Optimal Manpower Structure of DENMARK

\begin{tabular}{cccccccc}
\hline No. of workers & Level 1 & Level 2 & Level 3 & Level 4 & Level 5 & Level 6 & Total \\
\hline $\mathrm{t}=0$ & 350 & 250 & 150 & 120 & 80 & 50 & 1000 \\
$\mathrm{t}=1$ & 320 & 230 & 140 & 115 & 77 & 35 & 917 \\
$\mathrm{t}=2$ & 290 & 210 & 130 & 110 & 74 & 34 & 848 \\
$\mathrm{t}=3$ & 260 & 190 & 120 & 105 & 71 & 33 & 779 \\
$\mathrm{t}=4$ & 259 & 177 & 110 & 100 & 68 & 32 & 746 \\
$\mathrm{t}=5$ & 261 & 178 & 107 & 95 & 65 & 31 & 737 \\
$\mathrm{t}=6$ & 263 & 179 & 108 & 90 & 62 & 30 & 732 \\
$\mathrm{t}=7$ & 265 & 180 & 109 & 85 & 59 & 29 & 727 \\
$\mathrm{t}=8$ & 267 & 181 & 110 & 80 & 56 & 28 & 722 \\
$\mathrm{t}=9$ & 269 & 182 & 111 & 80 & 53 & 27 & 722 \\
$\mathrm{t}=10$ & 271 & 183 & 112 & 80 & 53 & 26 & 725 \\
$\mathrm{t}=11$ & 273 & 184 & 113 & 80 & 53 & 26 & 729 \\
$\mathrm{t}=12$ & 275 & 185 & 114 & 80 & 53 & 26 & 733 \\
\hline
\end{tabular}

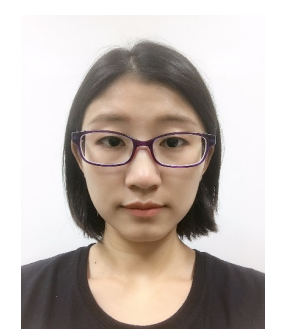

Yufei Sun received the Ph.D. degree from Curtin University, Perth, Australia, in 2015.

She is currently an associate research fellow with the School of Mathematical Sciences, Chongqing Normal University, Chongqing, China. Her research interests include optimal control, nonlinear programming, financial portfolio optimization.

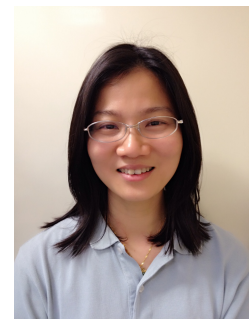

Grace Aw received the Ph.D. degree from Curtin University, Perth, Australia, in 2011.

She is currently a senior lecturer with the Department of Mathematics and Statistics, Curtin University, Perth, Australia. Her research interests include risk management, financial portfolio optimization and actuarial approach. 
TABLE VII

OPTIMAL HR STRATEGY OF CHINA

\begin{tabular}{|c|c|c|c|c|c|c|}
\hline Recruitment & Level 1 & Level 2 & Level 3 & Level 4 & Level 5 & Level 6 \\
\hline $\mathrm{t}=0$ & 287 & 182 & 88 & 0 & 0 & 0 \\
\hline $\mathrm{t}=1$ & 0 & 0 & 0 & 0 & 0 & 0 \\
\hline$t=2$ & 0 & 0 & 0 & 0 & 0 & 0 \\
\hline$t=3$ & 0 & 0 & 0 & 0 & 0 & 0 \\
\hline$t=4$ & 0 & 0 & 0 & 0 & 0 & 0 \\
\hline$t=5$ & 0 & 0 & 0 & 0 & 0 & 0 \\
\hline$t=6$ & 0 & 0 & 0 & 0 & 0 & 0 \\
\hline $\mathrm{t}=7$ & 0 & 0 & 0 & 0 & 0 & 0 \\
\hline $\mathrm{t}=8$ & 0 & 0 & 0 & 8 & 0 & 0 \\
\hline $\mathrm{t}=9$ & 0 & 0 & 0 & 8 & 0 & 0 \\
\hline$t=10$ & 0 & 0 & 0 & 8 & 0 & 1 \\
\hline$t=11$ & 0 & 0 & 0 & 5 & 0 & 1 \\
\hline$t=12$ & 0 & 0 & 0 & 0 & 0 & 0 \\
\hline Promotion & Level 1 & Level 2 & Level 3 & Level 4 & Level 5 & Level 6 \\
\hline $\mathrm{t}=0$ & 0 & 0 & 0 & 0 & 0 & 0 \\
\hline $\mathrm{t}=1$ & 0 & 0 & 0 & 0 & 0 & 0 \\
\hline$t=2$ & 0 & 0 & 0 & 0 & 0 & 0 \\
\hline$t=3$ & 0 & 0 & 0 & 0 & 0 & 0 \\
\hline$t=4$ & 0 & 0 & 0 & 0 & 0 & 0 \\
\hline$t=5$ & 0 & 0 & 0 & 0 & 0 & 0 \\
\hline$t=6$ & 0 & 0 & 0 & 0 & 0 & 0 \\
\hline$t=7$ & 0 & 0 & 0 & 0 & 0 & 0 \\
\hline $\mathrm{t}=8$ & 0 & 0 & 0 & 0 & 0 & 0 \\
\hline $\mathrm{t}=9$ & 0 & 0 & 0 & 0 & 3 & 0 \\
\hline$t=10$ & 0 & 0 & 0 & 0 & 3 & 0 \\
\hline$t=11$ & 0 & 0 & 0 & 0 & 3 & 0 \\
\hline$t=12$ & 0 & 0 & 0 & 0 & 0 & 0 \\
\hline Dismissal & Level 1 & Level 2 & Level 3 & Level 4 & Level 5 & Level 6 \\
\hline $\mathrm{t}=0$ & 0 & 0 & 0 & 0 & 0 & 0 \\
\hline$t=1$ & 0 & 0 & 0 & 0 & 0 & 14 \\
\hline $\mathrm{t}=2$ & 0 & 0 & 0 & 0 & 0 & 0 \\
\hline$t=3$ & 0 & 0 & 0 & 0 & 0 & 0 \\
\hline$t=4$ & 0 & 0 & 0 & 0 & 0 & 0 \\
\hline$t=5$ & 0 & 0 & 0 & 0 & 0 & 0 \\
\hline$t=6$ & 0 & 0 & 0 & 0 & 0 & 0 \\
\hline $\mathrm{t}=7$ & 0 & 0 & 0 & 0 & 0 & 0 \\
\hline $\mathrm{t}=8$ & 0 & 0 & 0 & 0 & 0 & 0 \\
\hline $\mathrm{t}=9$ & 0 & 0 & 0 & 0 & 0 & 0 \\
\hline$t=10$ & 0 & 0 & 0 & 0 & 0 & 0 \\
\hline $\mathrm{t}=11$ & 0 & 0 & 0 & 0 & 0 & 0 \\
\hline $\mathrm{t}=12$ & 0 & 0 & 0 & 0 & 0 & 0 \\
\hline
\end{tabular}

TABLE VIII

Optimal Manpower Structure of China

\begin{tabular}{cccccccc}
\hline No. of workers & Level 1 & Level 2 & Level 3 & Level 4 & Level 5 & Level 6 & Total \\
\hline $\mathrm{t}=0$ & 350 & 250 & 150 & 120 & 80 & 50 & 1000 \\
$\mathrm{t}=1$ & 607 & 412 & 228 & 115 & 77 & 35 & 1474 \\
$\mathrm{t}=2$ & 577 & 392 & 218 & 110 & 74 & 34 & 1405 \\
$\mathrm{t}=3$ & 547 & 372 & 208 & 105 & 71 & 33 & 1336 \\
$\mathrm{t}=4$ & 517 & 352 & 198 & 100 & 68 & 32 & 1267 \\
$\mathrm{t}=5$ & 487 & 332 & 188 & 95 & 65 & 31 & 1198 \\
$\mathrm{t}=6$ & 457 & 312 & 178 & 90 & 62 & 30 & 1129 \\
$\mathrm{t}=7$ & 427 & 292 & 168 & 85 & 59 & 29 & 1060 \\
$\mathrm{t}=8$ & 397 & 272 & 158 & 80 & 56 & 28 & 991 \\
$\mathrm{t}=9$ & 367 & 252 & 148 & 80 & 53 & 27 & 927 \\
$\mathrm{t}=10$ & 337 & 232 & 138 & 80 & 53 & 26 & 866 \\
$\mathrm{t}=11$ & 307 & 212 & 128 & 80 & 53 & 26 & 806 \\
$\mathrm{t}=12$ & 277 & 192 & 118 & 80 & 53 & 26 & 746 \\
\hline
\end{tabular}

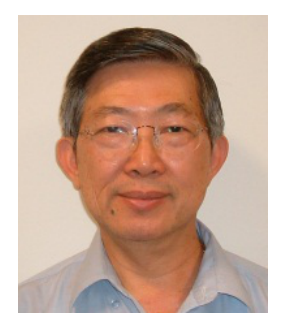

Kok Lay Teo received the Ph.D. degree from University of Ottawa, Ottawa, Canada.

He has been a John Curtin Distinguished Professor at Curtin University since 2011. He has published 5 books and over 500 journal papers. His editorial positions include serving as Editor-inChief of the Journal of Industrial and Management Optimization; Numerical Algebra, Control and Optimization; and Cogent Mathematics, and as a member of editorial board of a number of journals such as Automatica, Journal of Global Optimization, Journal of Optimization Theory and Applications, Optimization and Engineering, Discrete and Continuous Dynamic Systems, Optimization Letters, and Applied Mathematical Modelling. His research interests include both the theoretical and practical aspects of optimal control and optimization, and their practical applications such as in signal processing in telecommunications, and financial portfolio optimization. 


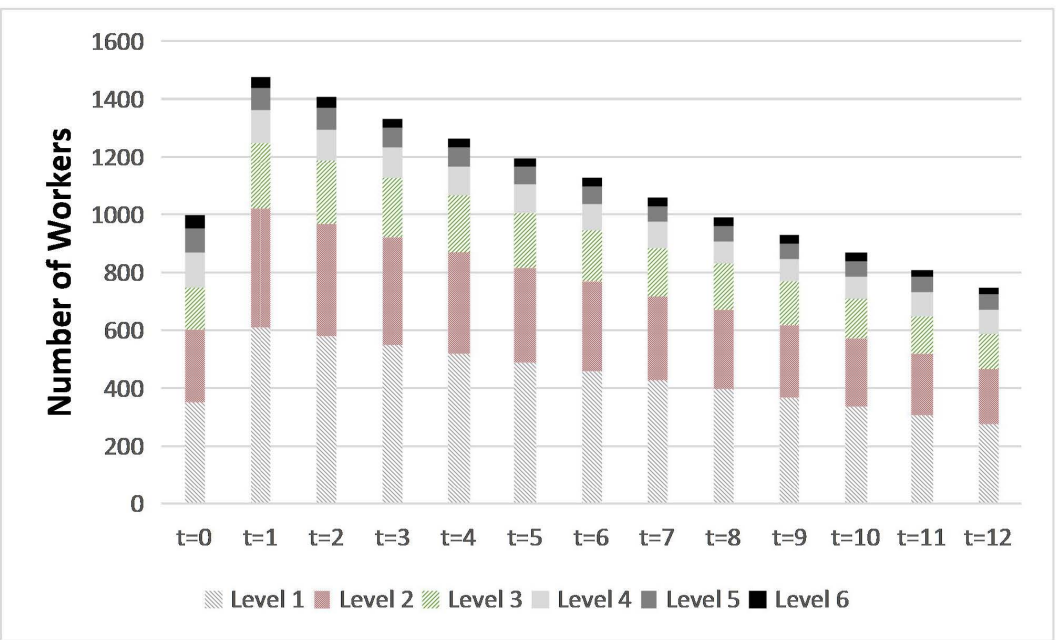

Fig. 3. Optimal Manpower Structure of China 